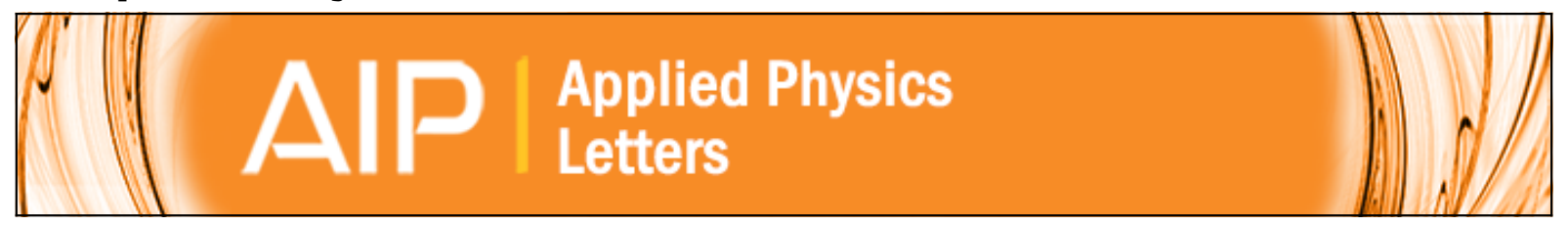

Domain wall broadening mechanism for domain size effect of enhanced piezoelectricity in crystallographically engineered ferroelectric single crystals

Wei-Feng Rao and Yu U. Wang

Citation: Applied Physics Letters 90, 041915 (2007); doi: 10.1063/1.2435584

View online: http://dx.doi.org/10.1063/1.2435584

View Table of Contents: http://scitation.aip.org/content/aip/journal/apl/90/4?ver=pdfcov

Published by the AIP Publishing

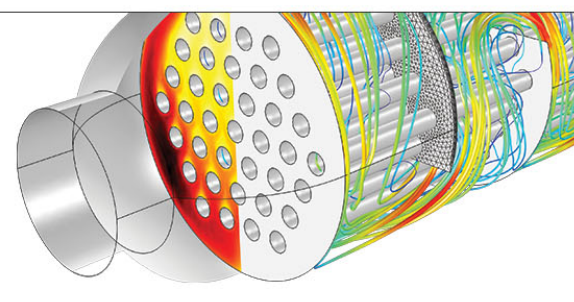




\title{
Domain wall broadening mechanism for domain size effect of enhanced piezoelectricity in crystallographically engineered ferroelectric single crystals
}

\author{
Wei-Feng Rao and Yu U. Wang ${ }^{\text {a) }}$ \\ Department of Materials Science and Engineering, Virginia Tech, Blacksburg, Virginia 24061
}

(Received 21 November 2006; accepted 17 December 2006; published online 25 January 2007)

\begin{abstract}
Computer modeling and simulation reveal a domain wall broadening mechanism that explains the domain size effect of enhanced piezoelectric properties in domain engineered ferroelectric single crystals. The simulation shows that, under electric field applied along the nonpolar axis of single crystal without domain wall motion, the domain wall broadens and serves as embryo of field-induced new phase, producing large reversible strain free from hysteresis. This mechanism plays a significant role in the vicinity of interferroelectric transition temperature and morphotropic phase boundary, where energy difference between stable and metastable phases is small. Engineered domain configuration fully exploits this domain wall broadening mechanism. (C) 2007 American Institute of Physics. [DOI: 10.1063/1.2435584]
\end{abstract}

High-performance piezoelectrics for sensors, actuators, and transducers are expected to exhibit large reversible strain free from hysteresis under electric field. However, the conventional strain mechanisms, such as electric field-induced domain wall motion and phase transition, are accompanied by large hysteresis. An important advance in piezoelectric research is the development of crystallographic domain engineering technique for ferroelectric single crystals. ${ }^{1,2}$ Single crystal with engineered domain configuration shows drastically enhanced, hysteresis-free strain versus electric field $(\epsilon-E)$ behavior along the nonpolar axis rather than the conventional polar axis. ${ }^{1,2}$ Recent experiments further discovered a domain size effect in the crystallographically engineered crystals, ${ }^{3,4}$ where the piezoelectric properties significantly increase with decreasing domain size. The domain size effect promises to further improve the electromechanical properties by engineering submicrometer-sized domains and develop environment friendly, lead-free piezoelectrics. $^{3,4}$

The minimization of $\epsilon$ - $E$ hysteresis in crystallographically engineered single crystal is achieved through the inhibition of electric field-induced domain wall motion. ${ }^{1-4}$ For engineered domain configuration, electric field is applied along the nonpolar axis of the single crystal, which forms equal angle to the polarization vectors of individual domains. All the domains are energetically and crystallographically equivalent with respect to the electric field in nonpolar direction, thus there is no driving force to domain wall motion, and electric field-induced intrinsic responses of these domains do not break such equivalency. As a result, tilted polarization remains in perfect head-to-tail patterns, and domain walls between intrinsically distorted domains are still charge-free crystallographic twin boundaries. Therefore, the piezoelectric properties of crystallographically engineered multidomain single crystal can be predicted from the properties of a single-domain single crystal along its nonpolar axis. Such single-domain intrinsic properties have been calculated and do exhibit piezoelectric anisotropy and enhanced ${ }^{\text {a) }}$ Author to whom correspondence should be addressed; electronic mail:
yuwang@mse.vt.edu response along the nonpolar axis. ${ }^{2,3,5}$ However, the calculated anisotropy only accounts for a small part of the experimentally measured property enhancement. ${ }^{3,4}$ Moreover, the calculation does not explain the recently observed domain size effect. ${ }^{3,4}$ Further investigation of the mechanism for crystallographic domain engineering is required. The purpose of this letter is to report a domain wall broadening mechanism that explains the enhanced piezoelectricity and domain size effect.

The domain size effect was discovered by directly correlating the measured piezoelectric properties to the observed domain configurations in barium titanate $\left(\mathrm{BaTiO}_{3}\right)$ and potassium niobate $\left(\mathrm{KNbO}_{3}\right)$ single crystals. ${ }^{3,4}$ Sophisticated thermo-electrical treatment has been developed to control the domain configurations and domain sizes. ${ }^{3,4}$ The micrometer-sized $(5.5-50 \mu \mathrm{m})$ domains allow direct domain observation and domain size measurement by polarized light microscopy. 3,4 Figure 1(a) shows the domain sizedependent piezoelectric properties $\left(d_{31}\right.$ and $\left.k_{31}\right)$ of [111]oriented tetragonal $\mathrm{BaTiO}_{3}$ single crystals. ${ }^{4}$ Based on the experimental data in Fig. 1(a), namely, $d_{31}=-97.8,-134.7$, $-180.1,-230.0 \mathrm{pC} / \mathrm{N}$ for average domain size (defined as domain width or thickness) $t=40,13.3,6.5,5.5 \mu \mathrm{m}$, respectively, and $d_{0}=-62.0 \mathrm{pC} / \mathrm{N}$ for single domain $(t=\infty),{ }^{4}$ we calculate the property increase for engineered multidomain configuration compared to single-domain state, i.e., $\Delta d_{31}$ $=\left|d_{31}-d_{0}\right|$. In Fig. 1(b), we plot $\Delta d_{31}$ versus the reciprocal domain size (proportional to domain wall density), $1 / t$. The data $\Delta d_{31}$ and $1 / t$ are well fitted into a straight line passing the origin $\left(\Delta d_{31}=0, t=\infty\right)$. The polarizing microscope observation shows that the engineered multidomain configurations are composed of tetragonal phase with $90^{\circ}$ domain walls ( $\{110\}$ twin boundaries). ${ }^{4}$ Since the domains of [100], [010], and [001] polarization orientations have equal energy in [111] electric field, the $90^{\circ}$ domain walls do not move. Thus, the quantity $\Delta d_{31}$ excludes both the intrinsic contribution $d_{0}$ from individual domains and the extrinsic contribution from domain wall motion. Therefore, the linear relationship between the property increase $\Delta d_{31}$ and the domain wall density $1 / t$ shown in Fig. 1(b) unambiguously attributes the piezoelectric property enhancement and domain size effect to 

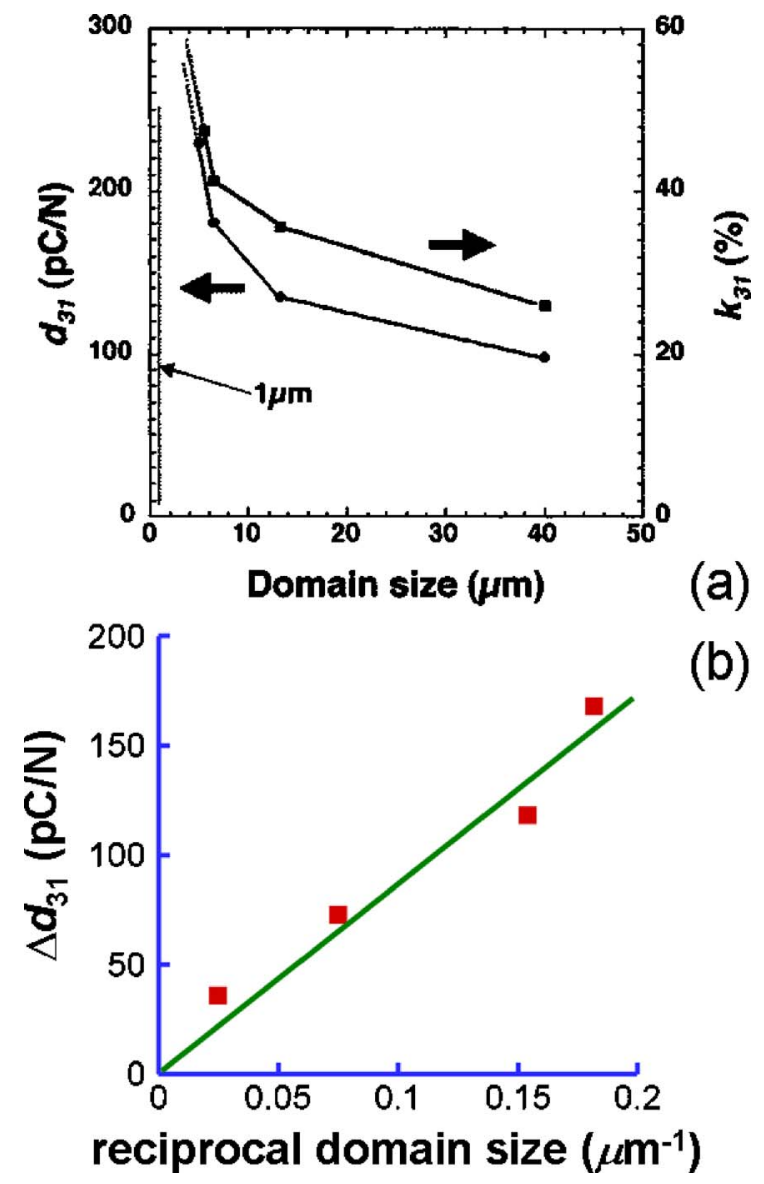

FIG. 1. (Color online) Domain size effect in [111]-oriented tetragonal barium titanate single crystals. (a) Experimentally measured piezoelectric properties vs domain size [reproduced with permission (Ref. 4)]. (b) A linear relationship between the property increase $\Delta d_{31}=\left|d_{31}-d_{0}\right|$ and reciprocal domain size (domain wall density), where the data (ם) are calculated from (a), and the straight line is obtained by fitting. The result unambiguously attributes the piezoelectric property enhancement and domain size effect to domain walls.

the domain walls themselves. A hypothesis of crystal lattice symmetry change in domain wall has been proposed to provide a qualitative explanation. ${ }^{6}$ However, direct experimental observation of domain wall behavior is not available, and a quantitative picture of the competition among several energy contributions in domain wall region needs to be explored, such as gradient energy of polarization rotation, elastic energy of lattice misfit, energy gap between metastable and stable phases, and external energy associated with applied electric field. In this work we employ computer modeling and simulation to study the underlying domain wall mechanism and clarify the role of domain walls under electric field along nonpolar axis.

The multidomain state of a ferroelectric single crystal is described by the polarization field $\mathbf{P}(\mathbf{r})$, whose total system free energy under electric field $\mathbf{E}^{\text {ex }}$ is given by ${ }^{7,8}$

$$
\begin{aligned}
F= & \int d^{3} r\left[f(\mathbf{P})+\frac{1}{2} \beta_{i j k l} \nabla_{i} P_{j} \nabla_{k} P_{l}-P_{k} E_{k}^{\mathrm{ex}}\right] \\
& +\frac{1}{2} \int \frac{d^{3} k}{(2 \pi)^{3}}\left[\frac{n_{i} n_{j}}{\varepsilon_{0}} \widetilde{P}_{i} \widetilde{P}_{j}^{*}+K_{i j k l} \widetilde{\varepsilon}_{i j}^{0} \widetilde{\varepsilon}_{k l}^{*}\right]
\end{aligned}
$$

where summation convention over repeated indices is implied. The function $f(\mathbf{P})$ is the nonequilibrium local bulk free energy density that defines the thermodynamic properties of stress-free homogeneous ferroelectric state and is formulated by Landau-Ginzburg-Devonshire polynomial expansion. ${ }^{9}$ The tensor $\beta_{i j k l}$ is the gradient coefficient that characterizes the energy contribution from polarization gradient in domain wall region, and $\nabla_{i}=\partial / \partial r_{i}$ is gradient operator. The $\mathbf{k}$-space integral gives the electrostatic and elastostatic energies of polarization distribution $\mathbf{P}(\mathbf{r})$ and misfit strain distribution $\boldsymbol{\epsilon}^{0}(\mathbf{r})$, respectively, where $\varepsilon_{0}$ is the permittivity of free space, $K_{i j k l}=C_{i j k l}-n_{m} C_{i j m n} \Omega_{n p} C_{k l p q} n_{q}, \Omega_{i k}=\left(C_{i j k l} n_{j} n_{l}\right)^{-1}, C_{i j k l}$ is the elastic modulus tensor, and $\mathbf{n}=\mathbf{k} / k$. The spontaneous lattice (misfit) strain is coupled to polarization through electrostriction coefficient tensor $Q_{i j k l}, \varepsilon_{i j}^{0}=Q_{i j k l} P_{k} P_{l}$. The functions $\widetilde{\mathbf{P}}(\mathbf{k})$ and $\tilde{\boldsymbol{\epsilon}}^{0}(\mathbf{k})$ are the Fourier transform of the fields $\mathbf{P}(\mathbf{r})$ and $\boldsymbol{\epsilon}^{0}(\mathbf{r})$, respectively, and the superscript " *" indicates the complex conjugate. The electric field-induced phase transition and domain evolution is characterized by the timedependent Ginzburg-Landau equation: ${ }^{7,8}$

$$
\frac{\partial P_{i}(\mathbf{r}, t)}{\partial t}=-L_{i j} \frac{\delta F}{\delta P_{j}(\mathbf{r}, t)}+\xi_{i}(\mathbf{r}, t)
$$

where $L_{i j}$ is the kinetic coefficient tensor, and $\xi_{i}(\mathbf{r}, t)$ is Gaussian-distributed Langevin noise term to account for the effect of thermal fluctuation. In this work, we use $\beta_{i j k l}$ $=\beta \delta_{i k} \delta_{j l}, L_{i j}=L \delta_{i j}$, and the experimentally determined polynomial coefficients $\left(\alpha_{i}, \alpha_{i j}, \alpha_{i j k}\right)$, elastic constants $\left(C_{i j k l}\right)$, electrostriction coefficients $\left(Q_{i j k l}\right)$ for lead zirconate titanate. $^{10}$

The computer simulation reveals a domain wall broadening mechanism, as shown in Fig. 2. We consider a single crystal of stable tetragonal phase with engineered domain configuration in electric field along the nonpolar [111] axis. The $90^{\circ}$ domain walls are also (110) twin boundaries. Figure 2(a) shows the equilibrium domain wall without electric field. The arrows represent the polarization vector distribution (the out-of-plane component is represented by color). When electric field is applied along the nonpolar [111] axis, the $90^{\circ}$ domain wall does not move due to the equivalent energetic state of the [100] and [010] tetragonal domains. However, as shown in Fig. 2(b), the domain wall broadens, where a layer of heavily stressed [111] rhombohedral domain is induced by the [111] electric field, which is coherently sandwiched between two adjacent stable tetragonal domains. Such an electric field-induced tetragonal $\rightarrow$ rhombohedral phase transition produces strain significantly larger than the intrinsic strain of tetragonal phase due to the polarization tilting under the same electric field, leading to enhanced piezoelectric response of the single crystal. As revealed by the simulation, the domain wall broadening behavior exhibits several important features. Firstly, the domain wall starts to broaden upon the application of electric field and gradually grows with increasing field, which produces enhanced piezoelectric response at low electric field. In this process, the $90^{\circ}$ domain wall serves as preexisting embryo of the fieldinduced rhombohedral phase. Secondly, the broadened domain wall shrinks reversibly with decreasing electric field, which produces hysteresis-free $\varepsilon-E$ curve. This reversibility is because the rhombohedral phase is metastable with respect to the stable tetragonal phase in the absence of electric field. Thirdly, the rhombohedral phase is epitaxially stressed by the tetragonal phase along the (110) interfaces. Although the elastic strain energy does not favor the formation of a layer of rhombohedral phase in the domain wall, the interplay 


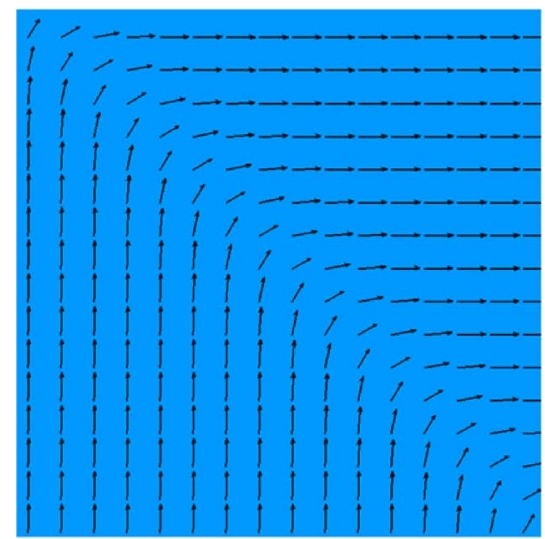

(a) no electric field

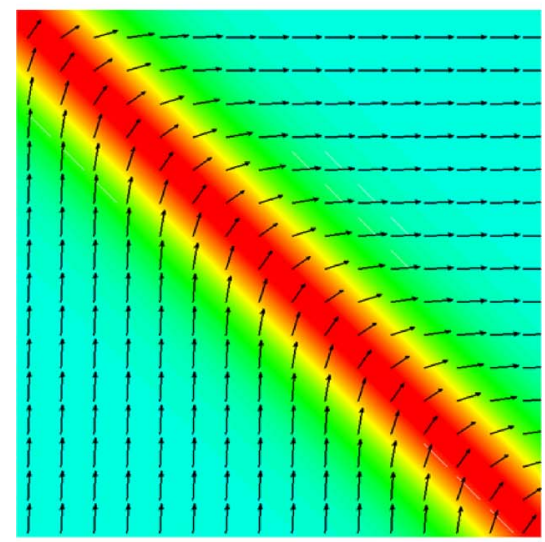

(b) [111] electric field

FIG. 2. (Color online) Computer simulation of domain wall broadening behavior under electric field along nonpolar axis. (a) Equilibrium $90^{\circ}$ domain wall and (110) twin boundary in tetragonal crystal without electric field. (b) Broadened domain wall composed of electric field-induced, heavily stressed [111] rhombohedral domain layer coherently sandwiched between tetragonal domains. The domain wall broadening mechanism explains the enhanced piezoelectric response along the nonpolar axis and the domain size (domain wall density) effect.

among various energetic contributions, as formulated in Eq. (1), favors domain wall broadening mechanism. Finally, since the contribution of phase transformation strain to the total crystal strain is proportional to the number of domain walls present in the crystal (i.e., domain wall density), the domain wall broadening mechanism leads to the domain size effect of enhanced piezoelectricity.

It is worth noting the similarity between lead-free $\mathrm{BaTiO}_{3}, \mathrm{KNbO}_{3}$ in the vicinity of interferroelectric transition temperatures and lead-based solid solutions $\mathrm{Pb}\left(\mathrm{Zr}_{1-x} \mathrm{Ti}_{x}\right) \mathrm{O}_{3}$, $\mathrm{Pb}\left[\left(\mathrm{Zn}_{1 / 3} \mathrm{Nb}_{2 / 3}\right)_{1-x} \mathrm{Ti}_{x}\right] \mathrm{O}_{3}, \quad \mathrm{~Pb}\left[\left(\mathrm{Mg}_{1 / 3} \mathrm{Nb}_{2 / 3}\right)_{1-x} \mathrm{Ti}_{x}\right] \mathrm{O}_{3}$ in the vicinity of morphotropic phase boundaries: there exist more than one ferroelectric phases (tetragonal, rhombohedral, orthorhombic) with small energy gap but large crystal lattice transformation strain in these perovskite-type ferroelectrics. An electric field-induced stable $\rightarrow$ metastable phase transition will produce a large strain associated with the crystal lattice rearrangement. However, in order to exploit the useful properties of the interferroelectric transition, two special requirements must be satisfied: (1) the electric field-induced phase transition must be reversible to achieve hysteresis-free $\varepsilon-E$ curve, and (2) the interferroelectric transition must be induced by sufficiently small electric field to achieve good piezoelectric response at low field regime. In order to meet these conditions, special domain configuration and electric field direction are required. As shown by our simulation (Fig. 2 ), the crystallographically engineered domain configuration and nonpolar electric field direction just satisfy these requirements. In this work, we considered $\mathrm{Pb}\left(\mathrm{Zr}_{1-x} \mathrm{Ti}_{x}\right) \mathrm{O}_{3}$ in the vicinity of morphotropic phase boundary. Figure 2(b) shows the [111] electric field-induced tetragonal $\rightarrow$ rhombohedral phase transition in domain wall region. Since the lead-free and lead-based perovskite-type ferroelectrics are characterized by the same Landau-Ginzburg-Devonshire polynomial with different material-specific expansion coefficients, our results also provide insight into the domain wall broadening mechanism in other systems. In particular, a [01] electric field-induced rhombic $\rightarrow$ rectangular transition is observed in $\mathrm{BaTiO}_{3}$ in the vicinity of interferroelectric transition temperature in a two-dimensional simulation, where rectangular phase nucleates at domain walls. ${ }^{11}$ Systematic investigation of the domain wall broadening mechanism and enhanced piezoelectric properties in different systems involving ferroelectric tetragonal, rhombohedral, and orthorhombic phases in the vicinity of transition temperatures and morphotropic phase boundaries will be reported in our future publication.

Finally, it is noteworthy that there are evidences showing that engineered nanodomain configuration exists in $\mathrm{Pb}\left[\left(\mathrm{Zn}_{1 / 3} \mathrm{Nb}_{2 / 3}\right)_{1-x} \mathrm{Ti}_{x}\right] \mathrm{O}_{3}$ and $\mathrm{Pb}\left[\left(\mathrm{Mg}_{1 / 3} \mathrm{Nb}_{2 / 3}\right)_{1-x} \mathrm{Ti}_{x}\right] \mathrm{O}_{3}$ single crystals. Intrinsic lattice parameter relationships ${ }^{12,13}$ and nanotwin diffraction analysis ${ }^{14}$ show that the monoclinic $M_{C}$ phase in domain engineered $\mathrm{Pb}\left[\left(\mathrm{Zn}_{1 / 3} \mathrm{Nb}_{2 / 3}\right)_{1-x} \mathrm{Ti}_{x}\right] \mathrm{O}_{3}$ and $\mathrm{Pb}\left[\left(\mathrm{Mg}_{1 / 3} \mathrm{Nb}_{2 / 3}\right)_{1-x} \mathrm{Ti}_{x}\right] \mathrm{O}_{3}$ is tetragonal nanotwins. Recent transmission electron microscopy experiment directly observes tetragonal twins of average domain size about $10 \mathrm{~nm},{ }^{15}$ which appears to be $M_{C}$ phase in diffraction and polarized light microscopy. Therefore, the domain wall broadening mechanism and domain size effect could play a role at nanoscale and be responsible for the superior piezoelectric properties along the nonpolar axis of these materials, which is under our investigation.

${ }^{1}$ S. E. Park and T. R. Shrout, J. Appl. Phys. 82, 1804 (1997).

${ }^{2}$ S. E. Park, S. Wada, L. E. Cross, and T. R. Shrout, J. Appl. Phys. 86, 2746 (1999).

${ }^{3}$ S. Wada, K. Muraoka, H. Kakemoto, T. Tsurumi, and H. Kumagai, Jpn. J. Appl. Phys., Part 1 43, 6692 (2004).

${ }^{4}$ S. Wada, K. Yako, H. Kakemoto, T. Tsurumi, and T. Kiguchi, J. Appl. Phys. 98, 014109 (2005).

${ }^{5}$ D. Damjanovic, J. Am. Ceram. Soc. 88, 2663 (2005).

${ }^{6}$ S. F. Liu, S. E. Park, H. Lei, L. E. Cross, and T. R. Shrout, Ferroelectrics 221, 169 (1999)

${ }^{7}$ S. Semenovskaya and A. G. Khachaturyan, J. Appl. Phys. 83, 5125 (1998).

${ }^{8}$ Y. L. Li, S. Y. Hu, Z. K. Liu, and L. Q. Chen, Appl. Phys. Lett. 78, 3878 (2001).

${ }^{9}$ A. F. Devonshire, Philos. Mag. 40, 1040 (1949).

${ }^{10}$ A. Amin, M. J. Haun, B. Badger, H. McKinstry, and L. E. Cross, Ferroelectrics 65, 107 (1985)

${ }^{11}$ R. Ahluwalia, T. Lookman, A. Saxena, and W. Cao, Appl. Phys. Lett. 84, 3450 (2004).

${ }^{12}$ Y. M. Jin, Y. U. Wang, A. G. Khachaturyan, J. F. Li, and D. Viehland, Phys. Rev. Lett. 91, 197601 (2003); J. Appl. Phys. 94, 3629 (2003).

${ }^{13}$ Y. U. Wang, Phys. Rev. B 73, 014113 (2006).

${ }^{14}$ Y. U. Wang, Phys. Rev. B 74, 104109 (2006).

${ }^{15}$ H. Wang, J. Zhu, N. Lu, A. A. Bokov, Z. G. Ye, and X. W. Zhang, Appl. Phys. Lett. 89, 042908 (2006). 